\title{
Molecular Simulation of Natural Gas Transport and Storage in Shale Rocks with Heterogeneous Nano-pore Structures
}

\author{
Shuai He, Yang Jiang, Jacinta C. Conrad, and Guan Qin* \\ Department of Chemical and Biomolecular Engineering, University of Houston, Houston, TX, \\ 77004, United States
}

Corresponding author. E-mail address: gqin@uh.edu

\begin{abstract}
Although natural gas is widely produced from shale, the mechanisms of natural gas transport in shale matrices remain poorly understood due to the complex chemical compounds of the matrices and the nanoscale pore size distribution. Using molecular simulations, we investigate natural gas transport and storage in nanopore networks. Carbonbased 3-D pore networks are generated from 2-D scanning electron microscopy (SEM) images of a shale rock using the Markov Chain Monte Carlo simulation method. We employ a grand canonical Monte Carlo (GCMC) simulation to calculate adsorption isotherms of natural gas in carbon-based 3-D pore networks, which can be fit by a Langmuir isotherm model. To investigate gas transport in the same structures, we insert an external driving force into non-equilibrium molecular dynamics (NEMD) simulations and find that Knudsen diffusion is the dominant transport mechanism in the pore networks. Although porosity and pore connectivity affect the natural gas diffusion in the pore networks, we typically observe a linear relationship between average molar flow rate through a cross-sectional area and the external driving force.
\end{abstract}

KEYWORDS: Digital rock, Molecular simulation, Langmuir adsorption, Knudsen diffusion 


\section{INTRODUCTION}

As conventional hydrocarbon resources are rapidly being depleted, shale gas has become increasingly important as a strategic energy supply for US energy independence and security (Ambrose et al., 2010; Civan, 2010; Collell et al., 2014; Darabi et al., 2012; Fathi et al., 2013; Javadpour, 2009; Mosher et al., 2013; Swami and Settari, 2012; Yiannourakou et al., 2013; Yuan et al., 2014). Shale gas is natural gas trapped in fine-grained sedimentary rock formations, which are usually characterized as anisotropic and heterogeneous porous media with very small pores. Consequently, the permeability of shale formations is extremely low and is typically of the order of magnitude of one hundred nanodarcies (Sakhaee-Pour and Bryant, 2012). The ultra-low permeability of shale formations presents a significant challenge in the production of shale gas in an economically viable fashion. Although horizontal drilling and multi-stage hydraulic fracturing stimulation make commercial shale gas production possible, the underlying mechanisms of gas transport in these highly confined media remain unclear and need to be fully understood for optimal production performance and reserve estimation (Collell et al., 2014).

There are two main challenges in identifying the underlying mechanisms of gas transport in shale reservoirs. First, petrophysical studies indicate that more than $60 \%$ of the pores in shale formations are micropores with a characteristic length scale of less than $30 \mathrm{~nm}$ (Darabi et al., 2012; Mosher et al., 2013). At this scale, the mean free path $\lambda$ of fluid molecules becomes comparable to the characteristic length $L$ of the flow channel. As one consequence, Darcy's equation $(v=-(k / \mu) \nabla P$, where $v$ is the mean fluid velocity, $\nabla P$ is the gradient of the pressure, $k$ is the permeability, and $\mu$ is the viscosity) cannot accurately describe transport at this scale because the continuum theory breaks down when the Knudsen number $K n=\lambda / L$ exceeds 
0.1 (Roy et al., 2003). Therefore, accurate descriptions of gas transport through nanoscale pores require experimental and/or numerical studies at these length scales. Second, besides the nanoscale pore size, shale matrices consist of various types of kerogen, clays, and other minerals that exhibit differences in porosity, tortuosity, and pore size distribution. Describing the intermolecular interaction between gas and boundary molecules in these heterogeneous pores, which heavily influences gas storage and transport behaviors, remains a significant challenge both experimentally and computationally. Methods to generate understanding of gas flow and storage mechanisms at the molecular level must address these challenges and are required to enhance shale gas production performance.

Towards this end, several recent studies have investigated gas storage and transport mechanisms in micropores using simple geometries and microporous model materials. Experimental measurements of high-pressure methane adsorption isotherms in porous media have been widely reported (Heller and Zoback, 2014; Ji et al., 2012; Rexer et al., 2014; Zhang et al., 2012). Numerically, sophisticated molecule structures (Katti et al., 2014; Zheng et al., 2014) and simple organic carbon molecule structures (Ambrose et al., 2010; Mosher et al., 2013) have been developed to replace kerogen molecules to investigate gas adsorption. Both experiments and simulations show that the adsorption obeys the Langmuir isotherm and that the adsorption capacity is associated with total organic carbon (TOC), organic matter type, thermal maturity, and clay minerals content (Heller and Zoback, 2014; Rexer et al., 2014; Zhang et al., 2012).

A variety of studies show that transport in nanoporous media exhibits a variety of noncontinuum features. Experiments demonstrate that fluid flow in carbon nanotubes differs from that predicted based on continuum hydrodynamics models, such as the Hagen-Poiseuille equation (Majumder et al., 2005). Similarly, the experimentally measured flow rate of water and 
air through nanoscale pores is measured to be several orders of magnitude faster than that predicted using continuum theory (Holt et al., 2006). Furthermore, slip boundary conditions in nanotube and planar flow have been widely reported (Chen et al., 2008; Kannam et al., 2011; Thomas and McGaughey, 2009). Finally, Knudsen diffusion and free molecular diffusion dominate flow mechanisms in nanochannels and nanotubes (Roy et al., 2003). As a complement to experimental studies, molecular simulation techniques, including dual control volume grand canonical molecular dynamics (DCV-GCMD) simulations (Boţan et al., 2013) and nonequilibrium molecular dynamics (NEMD) simulations (Wang et al., 2013, 2012), are widely employed to simulate permeation experiments. As one example, a computational study of gas transport in models of graphite-like 3-D porous materials shows that the porosity $(\phi)$, the ratio of the pore volume to the total volume of the rock, strongly affects permeability, with the lack of pore connectivity hindering gas transport at porosities of less than 0.2 (Firouzi and Wilcox, 2012).

Although these and other studies of simple geometries and microporous materials have provided fundamental insights into the mechanisms of fluid storage and transport in microscopic systems, they do not adequately capture the complexity or heterogeneity of shale gas matrices. These simplified models recreate neither the complex chemical composition (kerogen, clay, quartz, etc.) nor the complex pore connectivity found in shale. To generate more realistic models of natural shale, digital rock reconstruction (Wang and Pan, 2008), widely used in conventional reservoir modeling, can be used to recreate the detailed pore structure.

Generally, pore reconstruction techniques are necessary when 3D images with the required resolution are not easily acquired. Commonly used reconstruction methods can be divided into two classes. The first is the apparent-similarity method, which neglects the details of particle 
geometry and instead considers macroscopic properties (Li et al., 2005; Wang and Pan, 2008; Yang et al., 1996). An alternative method, digital rock reconstruction, focuses on the geometric details and aims to build more realistic structures by exploiting improvements in microscope instrumentation and in image mapping technology. In this technique, rock sections obtained by scanning microscopy methods, such as computed tomography (CT) or scanning electron microscopy (SEM), are digitally reconstructed. The detailed pore structure (porosity and connectivity) is recreated in the computational model. One algorithm commonly used in petroleum engineering and earth science research is the Markov Chain Monte Carlo (Wu et al., 2006, 2004) (MCMC) simulation, which recreates the 3-D microstructure based on thin 2-D images of rock samples obtained using SEM. Continuum fluid transport mechanisms have been widely studied in digital rock reconstructions (Boek and Venturoli, 2010; Manwart et al., 2002). By contrast, few studies have investigated transport mechanisms in the non-continuum flow regime in digital rock reconstructions, despite the increased complexity and verisimilitude of these models (Boţan et al., 2015). Studies of non-continuum transport in digital rock reconstructions are therefore expected to generate new insight into the transport mechanisms operating in nanoporous natural shale.

In this paper, we combine digital rock reconstruction and molecular simulation to investigate gas transport and storage and fluid-boundary molecular interactions in models of nanoporous shale matrices. In Section 2, we describe digital rock sample and simulation methods. We use an established method for 3-D pore network reconstruction, the Markov Chain Monte Carlo (MCMC) algorithm, to generate digital rock samples from 2-D SEM images of shale samples taken from the low Silurian Marine formation. To elucidate storage mechanisms, we use grand canonical Monte Carlo (GCMC) simulations to predict adsorption isotherms in carbon-based 
nanopore networks. To study gas transport, we perform NEMD simulations by inserting different external body force values in the system and subsequently correlate the average molar flow rate through the cross-sectional area to the external driving force. In Section 3, we discuss results for the adsorption isotherms and for gas transport. We find that the adsorption data can be fit by the Langmuir isotherm model and that Knudsen diffusion is the dominant transport mechanism. Conclusions and future work are discussed in Section 4.

\section{SIMULATION METHODS}

\subsection{Pore Structure Construction Method: MCMC Algorithm}

The MCMC method is used to model the spatially anisotropic and heterogeneous structure of multi-phase media (matrix, pore, etc.) based on 2-D thin section information (Wu et al., 2004). As shown in Fig. 1, the state of each particular voxel $\left(\mathrm{x}_{i j k}\right)$ is assigned a value of 0 or 1 , corresponding to pore or rock, respectively, and is conditionally dependent on the states of its neighboring voxels $N(i, j, k)$. The neighborhood $N(i, j, k)$ in the MCMC method, a new feature compared to previous methods (Geman and Geman, 1984), depends on the spatial dimensionality: $N(i, j, k)$ contains 2 neighbors in 1-D, 5 or 6 neighbors in 2-D, and 11, 12, or 15 neighbors in 3-D that are prior to the current position $\mathrm{x}_{i j k}$. The key advantage of the MCMC method is that, from some initial position $(i=1, j=1, k=1)$, the state of the remaining voxels only depends on the states of neighboring voxels that are previously determined; therefore, the MCMC method avoids iterative computation and speeds up the simulation procedure. The dependence of the target voxel on its neighbor is described by the conditional probability distribution, that is, $P\left(x_{i j k} \mid x_{l m n}:(l, m, n) \in N(i, j, k)\right)$. After the conditional probabilities of a specific voxel $(i, j, k)$ are 
calculated, a Monte Carlo simulation is used to sample the state of this voxel from its corresponding conditional probability distribution.

In our work, shale core samples are taken from the Longmaxi Formation that is a low Silurian marine formation in the Sichuan Basin, South China at depths of 6279-6385 ft. High resolution 2-D images of the shale rock samples are collected using SEM (Hitachi S-4800 Field Scanning Electron Microscope, Fig. 2a) and processed to form a binary microstructural data matrix (Fig. $2 \mathrm{~b}$ ) in which a digital pixel has an area of $1 \mathrm{~nm} \times 1 \mathrm{~nm}$.

From this data matrix, the transition probabilities between different neighbors (1-D and 2-D neighbors) are collected and stored by sampling 2-D neighborhoods and enumerating the different realizations for each configuration of the neighborhood (Wu et al., 2004). Next, the 3-D transition probability is obtained based on the 2-D transition probability. The probability that the first voxel $(i=1, j=1, k=1)$ is a pore is taken as the porosity in the SEM image, that is, the ratio of the number of pore pixels to the total number of pixels. Starting from the first voxel, a total of 64 million voxels $(400 \times 400 \times 400$ voxels $)$ are sampled sequentially using a Monte Carlo simulation. Following Zhao's method (Yao et al., 2013; Zhao et al., 2006), the average pore size distribution is shown in Fig. 3. Most pores have diameters of several nanometers, and the mean pore size is $2.35 \mathrm{~nm}$.

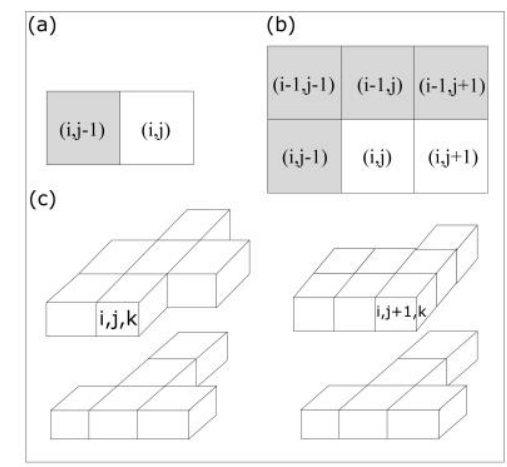

Fig. 1. Schematic illustration of the MCMC neighborhoods algorithm. (a) The 1-D 2-neighbor condition for updating voxel (i,j) based on (i,j-1). (b) The 2-D 5- and 6- neighbor conditions for updating two voxels (i,j) and 
$(\mathrm{i}, \mathrm{j}+1)$ simultaneously. (c) The 3-D 11- and 12- neighbor conditions for updating two voxels $(\mathrm{i}, \mathrm{j}, \mathrm{k})$ and $(\mathrm{i}, \mathrm{j}+1, \mathrm{k})$ simultaneously. From the initial point $(\mathrm{i}=1, \mathrm{j}=1, \mathrm{k}=1)$, the voxels in the first row are conditionally determined by the 1-D neighbors. Next, voxels in the same plane $(i, j, k=1)$ are conditionally determined by the 2-D neighbors, and the remaining voxels are determined by the $3-\mathrm{D}$ neighbors.
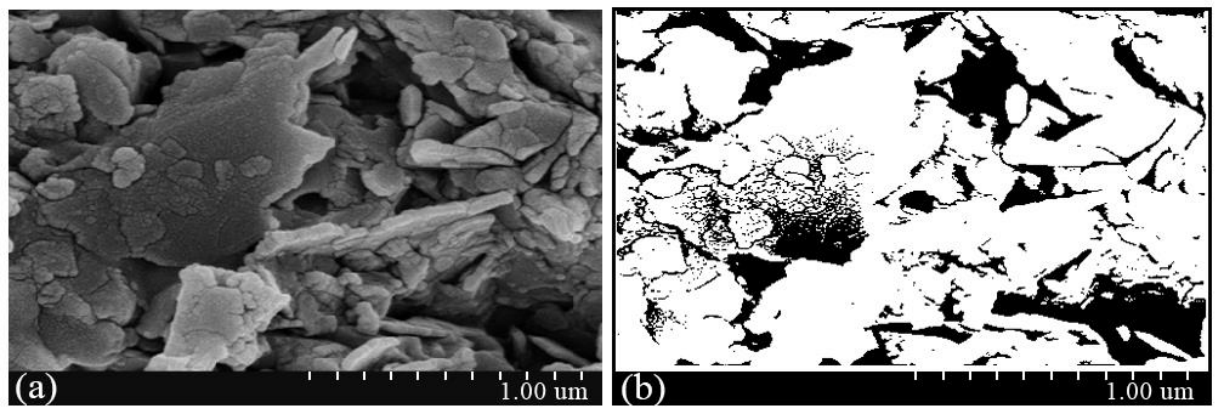

Fig. 2. (a) Scanning electron micrograph of shale organic matter. (b) Two-value reconstruction of the SEM image in (a), in which values of 0 and 1 correspond to pore and rock voxels, respectively.

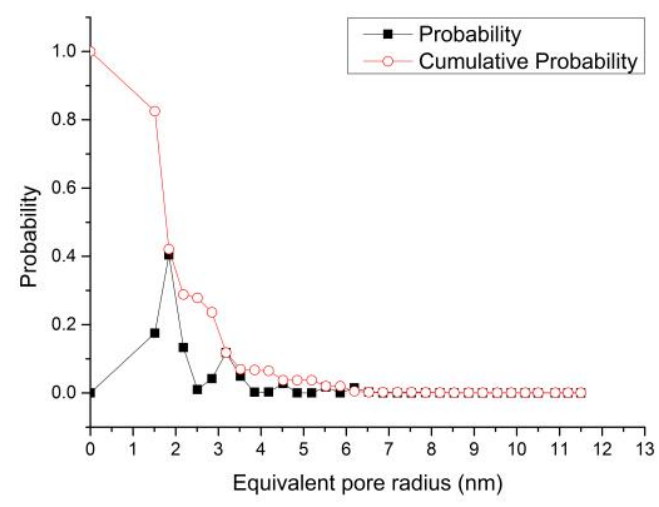

Fig. 3. Pore size distribution and cumulative pore size distribution of the 3-D digital rock. The cumulative pore size distribution is the probability that radii of pores are smaller than a given equivalent radius.

\subsection{Pore Networks Sampling and Molecular Structure Implementation}

Three different pore networks with different porosities are extracted from one reconstructed digital rock based on the MCMC algorithm and are used in molecular simulations. Each pore structure consists of $600=10 \times 10 \times 6(x, y, z)$ connected voxels. The porosities of the three porous media are taken as the ratio of the number of the pore voxels to the total number of voxels in each structure. The surface area is taken as the number of pore-solid interfaces. 
Periodic boundary conditions are used in the determination of the surface area. Table 1 shows the porosities and surface area in the three pore networks.

Table 1. Porosities and surface areas in three pore networks generated from the digital rock image.

\begin{tabular}{ccc}
\hline Sample & Porosity $(\%)$ & Surface area $\left(\mathrm{nm}^{2}\right)$ \\
1 & $16.7(100 / 600)$ & 322 \\
2 & $25.0(150 / 600)$ & 476 \\
3 & $39.2(235 / 600)$ & 640 \\
\hline
\end{tabular}

After the value of each voxel ( 0 or 1$)$ in the pore networks is generated using the MCMC method, molecular structures are inserted into the pore network. In pore voxels, we insert methane molecules to represent natural gas. In rock voxels, we model the effect of organic matter by inserting a related molecular structure. A major constituent of the organic matter found in sedimentary rocks is kerogen, a mixture of high molecular weight organic compounds, which can exhibit a wide variety of compositions depending on the source shale formation (Collell et al., 2014; Heller and Zoback, 2014; Orendt et al., 2013) and therefore cannot be readily represented with one (or even a few) molecular structure(s). Instead, as the initial test of our new hybrid simulation approach, we replace the rock voxels with simple center cubic (SCC) carbon structures because carbon atoms play a significant role in the interactions between gas and solid molecules in shale formations. In our study, the length of a voxel is $1 \mathrm{~nm}$ in each direction $(x, y, z)$; the molecular diameter of carbon is $0.34 \mathrm{~nm}$. We therefore insert $27=3 \times 3 \times 3(x, y, z)$ carbon atoms in each rock voxel to represent the organic carbon structure. Methane cannot penetrate the carbon-filled rock voxels and only transports through pore voxels. Depending on the number of rock voxels in each pore network, $13,500,12,150$, and 9,855 carbon atoms are inserted. 
Methane and carbon atoms are assumed to act as hard spheres and are represented using cutoff Lennard-Jones (LJ) intermolecular potentials; the interactions are characterized by the effective LJ size and energy parameters, $\sigma$ and $\varepsilon$, respectively. The methane-carbon interaction is modeled by taking the average of the two interactions based on the Lorentz-Berthelot mixing rule (Mosher et al., 2013). The parameters used in the simulations are shown in Table 2.

Table 2. Lennard-Jones interaction parameters used in simulation calculations.(Mosher et al., 2013)

\begin{tabular}{ccc}
\hline LJ intermolecular potential & $\varepsilon(\mathrm{Kcal} / \mathrm{mol})$ & $\sigma(\mathrm{nm})$ \\
Methane-methane & 0.294 & 0.373 \\
Carbon-carbon & 0.056 & 0.3405 \\
Methane-carbon & 0.128 & 0.3567 \\
\hline
\end{tabular}

The cut-off Lennard-Jones potential is given by

$$
U(r)=\left\{\begin{array}{lr}
U_{L J}(r)-U_{L J}\left(r_{c}\right), r<r_{c} \\
0, & r>r_{c}
\end{array}\right.
$$

where $U_{L J}(r)$ is the standard two-body LJ potential,

$$
U_{L J}(r)=4 \varepsilon\left[\left(\frac{\sigma}{r}\right)^{12}-\left(\frac{\sigma}{r}\right)^{6}\right]
$$

Here, $r$ is the distance between the interacting pair and $r_{c}$ is the cut-off distance. The cut-off distances of carbon and methane molecules are set as $r_{c}=12 \AA$. Because the methane has a relatively weak octupole moment (Martin and Siepmann, 1998), it is treated as a one-center Lennard-Jones sphere; neither long-range interactions nor electrostatic forces were included in this simulation.

\subsection{Molecular Simulation Setup}

To study the gas storage mechanism, we use a Monte Carlo simulation with a grand canonical ensemble, in which temperature (T), volume $(\mathrm{V})$, and chemical potential $(\mu)$ are held constant. In this simulation, carbon-based pore networks interact with an ideal gas reservoir with a specific 
chemical potential. Methane molecules in the gas reservoir are adsorbed in the porous media until equilibrium is obtained. The carbon atoms representing rock voxels are constrained at their initial position by adding a harmonic attractor. Periodic boundary conditions are applied in all three directions, and the porous medium initially contains no methane molecules. Simulation trial moves consist of molecular displacement, molecule insertion, and deletion. In total, Monte Carlo simulations consisting of approximately one million moves at each of six different temperatures ranging from $300-420 \mathrm{~K}$ are carried out. We count the number of methane molecules adsorbed in the carbon-based pore network when the system reaches equilibrium as a function of the chemical potential. The relationship between the chemical potential and the gas pressure in the gas reservoir is given by

$$
\frac{\mu}{k}=T \ln \left(\frac{P \Lambda^{3}}{k T}\right)
$$

where $k$ is Boltzmann's constant, $T$ is the temperature, $P$ is the pressure, and $A$ is the thermal de Broglie wavelength. A MCCCS (Monte Carlo for Complex Chemical Systems) Towhee molecular simulator is used for the simulation process.

To investigate methane transport behavior in the porous network, non-equilibrium molecular dynamics simulations with different external driving forces are carried out. Two methane molecules are inserted into each pore voxel so that the molar density $n_{v}$ is initially constant (two molecules $\left./ \mathrm{nm}^{3}\right)$ in each pore network. At this $\mathrm{n}_{\mathrm{v}}$, the mean-free path $\lambda=\left(\sqrt{2} \pi d^{2} n_{v}\right)^{-1}$, calculated from kinetic theory (Chapman and Cowling, 1970) using the molecular diameter of methane $d=$ $0.373 \mathrm{~nm}$ and a pore voxel volume of $(1 \mathrm{~nm})^{3}$, is $\lambda \approx 0.8 \mathrm{~nm}$. We take the characteristic length scale as the mean pore diameter, $\mathrm{L}=4.70 \mathrm{~nm}$, and calculate a Knudsen number $\mathrm{Kn}=\lambda / \mathrm{L}=0.2$. Because $\mathrm{Kn}>0.1$, the flow is in the non-continuous regime. 
To initialize the sample, we perform an equilibrium molecular dynamics simulation with a constant NVT ensemble (i.e., in the canonical ensemble). The temperature is set at $300 \mathrm{~K}$ and is controlled by the Berendsen thermostat (Berendsen et al., 1984) algorithm with a time scale parameter of $100 \mathrm{fs}$. The duration of the initial run is approximately $10 \mathrm{ps}$ with a time step of 1 fs. After the initial run, an energetic minimization process is applied by iteratively adjusting the atomic coordinates to reach the local energetic minimum. Next, a non-equilibrium molecular dynamics simulation is carried out by inserting an external body force in the $x$-direction using the Varlet integration algorithm in an NVT $(\mathrm{T}=300 \mathrm{~K})$ ensemble coupled with the Nose-Hoover thermostat algorithm (Frenkel and Smit, 2002). The relaxation time in the Nose-Hoover algorithm is set at $100 \mathrm{fs}$. The time step is selected as $1 \mathrm{fs}$, and the total simulation time for each run is $80-100$ ps. All MD simulations are performed using LAMMPS (a large-scale atomic/molecular massively parallel simulator) (Plimpton, 1995).

\subsection{Flow Rate Calculations}

In complex pore networks, we calculate the relationship between the external body force and the total molar flow rate through a cross-sectional layer. Fig. 4 illustrates a single cross-sectional layer perpendicular to the flow (positive $x$ ) direction.

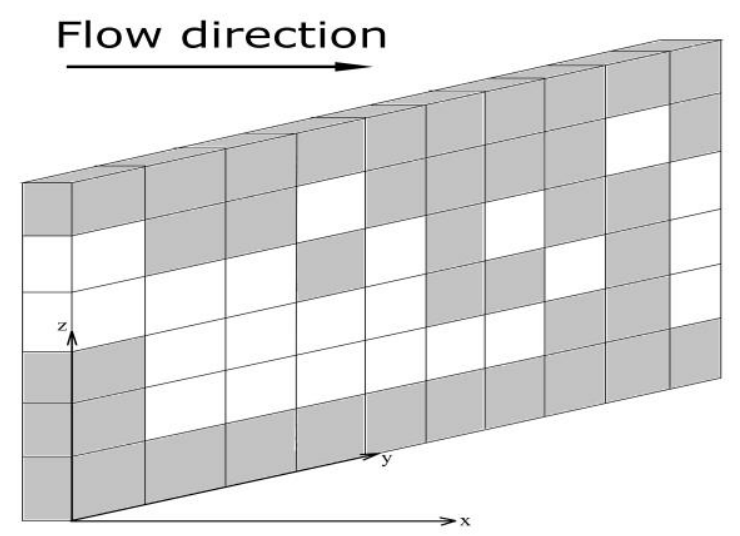

Fig. 4. Schematic illustrating the cross-sectional structure of a complex pore network perpendicular to the $x$ direction (the direction of flow). Gray and white represent rock and pore voxels, respectively. 
The molar flow rate through the $\mathrm{i}^{\text {th }}$ pore voxel $M F_{i}$ is given by

$$
M F_{i}=(\rho \bar{v})_{x i} A_{\text {pore }}=\frac{N_{i}}{V_{\text {pore }}} \frac{\sum_{j=1}^{N_{i}} v_{x j}}{N_{i}}=\frac{A_{\text {pore }}}{V_{\text {pore }}} \sum_{j=1}^{N_{i}} v_{x j}=\frac{1}{L_{\text {pore }}} \sum_{j=1}^{N_{i}} v_{x j}
$$

where $\rho$ represents the molar density, $A_{\text {pore }}$ is the cross-sectional area of a voxel, $V_{\text {pore }}$ is the volume of a voxel, and $L_{\text {pore }}=V_{\text {pore }} / A_{\text {pore }}$ is the length of the voxel. The mean velocity in the $x$ direction $\overline{v_{x i}}$ is calculated as the average of all molecular velocities in the $x$-direction $v_{x j} . N_{i}$ represents the number of methane molecules in each pore voxel. By summing all molar flow rates in each pore voxel in the cross-sectional layer, the total molar flow rate $M F$ through a given cross-section is calculated as

$$
M F=\sum_{i=1}^{N_{p}} M F_{i}=\frac{1}{L_{\text {pore }}} \sum_{i=1}^{N_{p}} \sum_{j=1}^{N_{i}} v_{x j}
$$

where $N_{p}$ represents the number of pore voxels in each cross-sectional layer.

\section{RESULTS AND DISCUSSION}

\subsection{Adsorption Isotherms in Pore Networks}

The adsorption isotherms of methane in the three pore networks at a temperature of $300 \mathrm{~K}$ are of the Langmuir type, as shown in Fig. 5. The amount of adsorbed gas increases with pressure at low pressures less than $100 \mathrm{MPa}$ and approaches a maximum adsorption at higher pressures. We fit the isotherms in Fig. 5 using the Langmuir isotherm equation, $N / N_{\max }=K P /(1+K P)$, and extract two parameters, $N_{\max }$ and $K$, as reported in Table 3. 


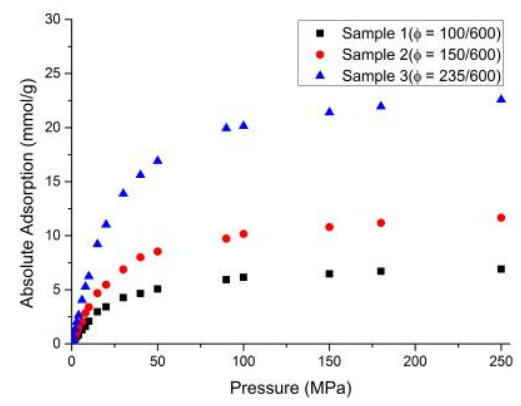

Fig. 5. Adsorption isotherms of three pore networks at $T=300 \mathrm{~K}$. $\phi$ indicates the porosity of the pore network.

Table 3. Langmuir parameters of methane adsorbed in the three pore networks at $300 \mathrm{~K}$, obtained from a fit to the Langmuir isotherm equation.

\begin{tabular}{ccccc}
\hline Sample & Porosity $\phi(\%)$ & $\begin{array}{r}\text { Surface area } \\
\left(\mathrm{nm}^{2}\right)\end{array}$ & $N_{\max }(\mathrm{mmol} / \mathrm{g})^{a}$ & $K\left(\times 10^{-3}, \mathrm{MPa}^{-1}\right)^{b}$ \\
1 & 16.7 & 322 & $7.69[7.52,7.86]$ & $38.03[35.31,40.75]$ \\
2 & 25.0 & 476 & $12.92[12.66,13.18]$ & $36.54[34.20,38.89]$ \\
3 & 39.2 & 640 & $25.58[24.90,26.25]$ & $36.34[33.31,39.38]$
\end{tabular}

${ }^{a}$ Brackets indicate the $95 \%$ confidence interval for each fitting parameter, $\left(N_{\max }\right.$ and $\left.K\right)$

${ }^{b}$ The Langmuir equation has the form $\frac{N}{N_{\max }}=\frac{K P}{1+K P}$, where $N$ and $P$ represent the absolute adsorption amount and pressure, respectively.

$K$ is the adsorption equilibrium constant, which is related to the changes in energy during the adsorption process. We find that the values of $K$ are similar for all three networks. In our model, carbon atoms are used to replace organic matter, and the carbon-methane interaction is independent of the geometric details of the pore networks; we therefore expect that $K$ should not depend strongly on porosity or connectivity. $N_{\max }$ represents the maximum adsorption capacity for each pore network. We find that $N_{\max }$ monotonically increases with increasing porosity and/or surface area, but the dependence of $N_{\max }$ on these parameters is not linear. This finding indicates that the gas storage mechanism is complex and may depend on multiple geometrical properties of the pore network. 
Table 4. Comparison of the adsorption capacities of the three pore networks at a temperature of $300 \mathrm{~K}$.

\begin{tabular}{cccccc}
\hline Sample & $\phi(\%)$ & $\begin{array}{c}\text { Surface area } \\
\left(\mathrm{nm}^{2}\right)\end{array}$ & $\begin{array}{c}N_{\max } \\
(\mathrm{mmol} / \mathrm{g})\end{array}$ & $\begin{array}{c}N_{\max } / \phi^{a} \\
(\mathrm{mmol} / \mathrm{g})\end{array}$ & $\begin{array}{c}N_{\max } / S A^{b} \\
\left(\mu \mathrm{mol} /\left(\mathrm{g} \cdot \mathrm{nm}^{2}\right)\right)\end{array}$ \\
1 & 16.7 & 322 & 7.69 & 46.13 & 23.88 \\
2 & 25.0 & 476 & 12.92 & 51.68 & 27.14 \\
3 & 39.2 & 640 & 25.58 & 65.31 & 39.97 \\
${ }^{a} \mathrm{~N}_{\max } / \phi$ : the ratio of maximum adsorption amount to the porosity of each pore network. \\
${ }^{b} \mathrm{~N}_{\max } / S A$ : the ratio of maximum adsorption amount to the surface area. \\
\hline
\end{tabular}

Adsorption isotherms of methane in the three pore networks at six different temperatures are shown in Fig. 6, and the Langmuir parameters obtained from fits for all networks are shown in Table 5. At fixed pressure, the absolute adsorption monotonically decreases as the temperature increases. We find that $N_{\max }$ is nearly independent of temperature, whereas $K$ decreases as the temperature increases. Because $N_{\max }$ is related only to the total number of active sites on the carbon surface, it should not vary with temperature. $K$, the adsorption equilibrium constant, is the ratio of the rate constant of adsorption to the rate constant of desorption (Atkins and de Paula, 2006). As the temperature is increased, more adsorbed gas will desorb from sites and free methane molecules will have more difficulty attaching on the carbon surface. This argument suggests that as the temperature is increased the rate of adsorption decreases while the rate of desorption increases, leading to a decrease in the equilibrium constant $K$.

Table 5. Langmuir parameters of the three pore networks, obtained at different temperatures with $95 \%$ confidence intervals.

\begin{tabular}{ccccc}
\hline Sample $^{a}$ & Temp. (K) & 300 & 315 & 330 \\
\hline \multirow{2}{*}{1} & $\mathrm{~N}_{\max }(\mathrm{mmol} / \mathrm{g})$ & $7.688[7.516,7.859]$ & $7.625[7.464,7.787]$ & $7.636[7.488,7.784]$ \\
& $\mathrm{K}\left(\times 10^{-3}, \mathrm{MPa}^{-1}\right)$ & $38.03[35.31,40.75]$ & $33.54[31.34,35.74]$ & $30.15[28.39,31.91]$
\end{tabular}




\begin{tabular}{|c|c|c|c|c|}
\hline \multirow[b]{2}{*}{2} & $\mathrm{~N}_{\max }(\mathrm{mmol} / \mathrm{g})$ & $12.92[12.66,13.18]$ & $12.83[12.53,13.13]$ & $12.49[12.25,12.73]$ \\
\hline & $\mathrm{K}\left(\times 10^{-3}, \mathrm{MPa}^{-1}\right)$ & $36.54[34.20,38.89]$ & $32.21[29.90,34.51]$ & $30.47[28.68,32.25]$ \\
\hline \multirow{2}{*}{3} & $\mathrm{~N}_{\max }(\mathrm{mmol} / \mathrm{g})$ & $25.58[24.90,26.25]$ & $25.43[24.79,26.06]$ & $25.45[24.94,25.96]$ \\
\hline & $\mathrm{K}\left(\times 10^{-3}, \mathrm{MPa}^{-1}\right)$ & $36.34[33.31,39.38]$ & $31.97[29.53,34.41]$ & $28.38[26.70,30.07]$ \\
\hline Sample & Temp. (K) & 360 & 390 & 420 \\
\hline \multirow[b]{2}{*}{ ( } & $\mathrm{N}_{\max }(\mathrm{mmol} / \mathrm{g})$ & $7.545[7.362,7.728]$ & $7.302[6.835,7.769]$ & $7.345[7.183,7.508]$ \\
\hline & $\mathrm{K}\left(\times 10^{-3}, \mathrm{MPa}^{-1}\right)$ & $25.27[23.51,27.03]$ & $23.27[19.09,27.44]$ & $18.73[17.64,19.82]$ \\
\hline \multirow{2}{*}{2} & $\mathrm{~N}_{\max }(\mathrm{mmol} / \mathrm{g})$ & $12.49[12.29,12.69]$ & $12.38[12.22,12.55]$ & $11.91[11.44,12.37]$ \\
\hline & $\mathrm{K}\left(\times 10^{-3}, \mathrm{MPa}^{-1}\right)$ & $24.15[23.06,25.24]$ & $20.88[20.14,21.62]$ & $18.48[16.59,20.37]$ \\
\hline & $\mathrm{N}_{\max }(\mathrm{mmol} / \mathrm{g})$ & $24.80[24.35,25.24]$ & $24.71[24.31,25.10]$ & $23.99[23.73,24.25]$ \\
\hline & $\mathrm{K}\left(\times 10^{-3}, \mathrm{MPa}^{-1}\right)$ & $23.97[22.75,25.19]$ & $19.91[19.07,20.76]$ & $17.95[17.45,18.46]$ \\
\hline
\end{tabular}

${ }^{a}$ Sample numbers correspond to those defined in Table 1.

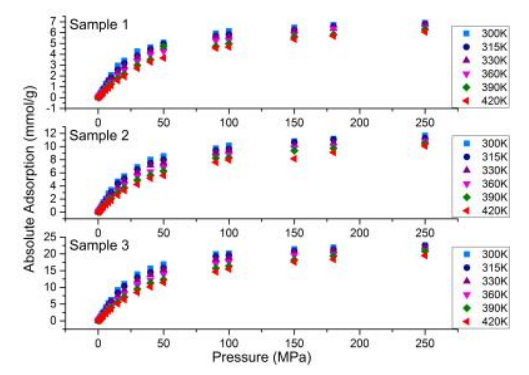

Fig. 6. Adsorption isotherms of methane in three pore networks at temperatures ranging from $300-420 \mathrm{~K}$.

The dependence of the equilibrium constant on temperature is described by the van't Holf equation (Atkins and de Paula, 2006),

$$
\frac{d \ln (K)}{d T}=\frac{\Delta H}{R T^{2}}
$$

in which $\Delta \mathrm{H}$ represents the adsorption enthalpy and $\mathrm{R}$ represents the ideal gas constant. From linear regressions of $\ln (\mathrm{K})$ as a function of $1 / \mathrm{T}$, shown in Fig. 7, we obtain the slope of the fit line, $-\Delta H / R$, and thus calculate the enthalpy, as shown in Table 6 . In each of the three pore 
networks, we obtain a negative enthalpy, indicating that the adsorption process is exothermic. As the surface area of the pore network is increased, more methane molecules adsorb on the carbon surface and more heat is released, leading to low enthalpy.

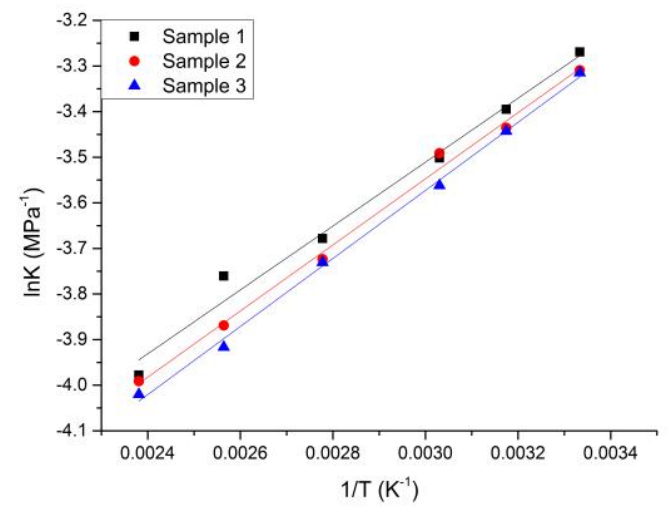

Fig. 7. Adsorption equilibrium constant $\ln (\mathrm{K})$ as a function of temperature $1 / \mathrm{T}$. Lines indicate linear regression.

Table 6. Linear regression of $\ln (K)=-\Delta H / R T+C$ from Equation 6. Here, enthalpy $(\Delta \mathrm{H})$ is assumed to be constant over the temperature range $(300-420 \mathrm{~K})$.

\begin{tabular}{cccccc}
\hline Sample & $-\frac{\Delta H}{R}$ & $\begin{array}{c}95 \% \text { confidence } \\
\text { interval }\end{array}$ & $C$ & $\begin{array}{c}95 \% \text { confidence } \\
\text { interval }\end{array}$ & $\mathrm{R}^{2}$ \\
1 & 700.6 & {$[586.3,814.9]$} & -5.61 & {$[-5.94,-5.28]$} & 0.9864 \\
2 & 724.9 & {$[655.3,794.5]$} & -5.72 & {$[-5.92,-5.52]$} & 0.9952 \\
3 & 746.3 & {$[697.3,795.2]$} & -5.81 & {$[-5.95,-5.67]$} & 0.9978 \\
\hline
\end{tabular}

\subsection{Transport Behavior in Pore Networks}

Fig. 8 shows the time-averaged molar flow rate as a function of layer number within the pore networks in four different external body forces through Sample 1. The molar flow rates in different layers fluctuate across a wide range. To quantify the magnitude of fluctuations, we 
calculate the percent deviation of the flow rate in each layer from the mean, that is, $f=\frac{M F_{\text {each }}-M F_{\text {mean }}}{M F_{\text {mean }}} \times 100 \%$

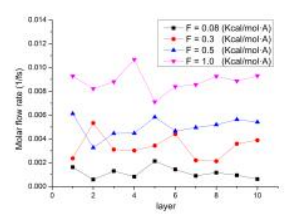

Fig. 8. Molar flow rate of methane as a function of layer (with 10 total layers created based on the dimension of MCMC digital rocks) in Sample 1 and of the external driving force applied in the pore networks. The units of external force $(\mathrm{Kcal} / \mathrm{mol} \cdot \AA)$ and molar flow rate $(1 / \mathrm{fs})$ are consistent with the units in LAMMPS.

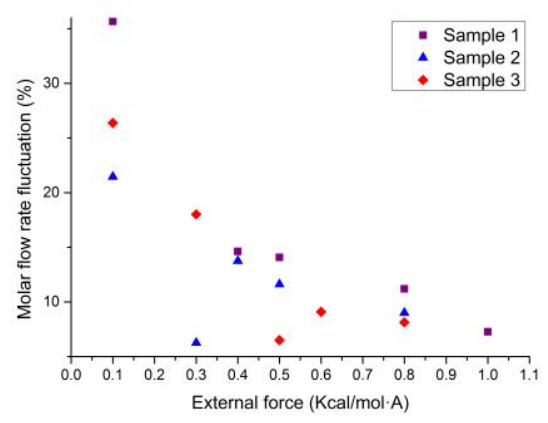

Fig. 9. Average molar flow rate through the three networks as a function of external driving force. The fluctuation is calculated as the percentage deviation of the molar flow rate in each layer from the mean.

Across all samples and all external forces investigated, the average fluctuation of the molar flow rate in each layer typically decreases as the external driving force is increased, as shown in Fig. 9. This result indicates that molecular (or Knudsen) diffusion dominates the transport behavior in these nanoscale pore networks. Further evidence for diffusion-dominated transport arises when a weak external force is applied to drive flow through the pore networks. When the external force is weak $(\mathrm{F}=0.03 \mathrm{Kcal} / \mathrm{mol} \cdot \AA)$, the molar flow rate values are typically larger and more positive than those in the equilibrium state (in which there is no external driving force), which fluctuate around zero (Fig. 10). This result confirms that the external driving force affects 
the molecular trajectories such that the majority of the molecules move in the direction of the external force. This effect may be insignificant compared to the molecular diffusion, however, if the external force is not sufficiently strong. Indeed, the molar flow rate in Sample 2 is negative in one layer (layer 7), indicating that the average flow direction in that layer opposes the direction of the weak external driving force.

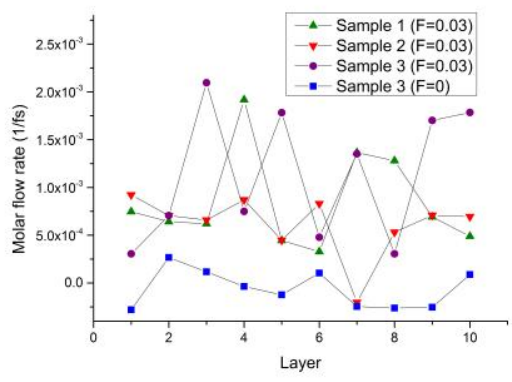

Fig. 10. Molar flow rates as a function of layer in three pore networks with weak external body force compared to that in the equilibrium molecular simulation (no external force) in Sample 3. In the equilibrium molecular simulation, the molar flow rate in different layers fluctuates around zero, consistent with molecular diffusion as the dominant transport mechanism.

Fig. 11 shows the averaged molar flow rate as a function of the external body force in each pore network; to eliminate any possible end effects within each pore network, the average molar flow rate through the pore network is calculated as the mean molar flow rate in layers $3-8$. The average molar flow rate is linearly proportional to the external body force. A similar linear dependence was reported in Ref. 12, in which the molar flow rate $M F$ experimentally measured in nanopores was proportional to the pressure drop via

$$
\frac{M F}{\varepsilon A}=D_{K} \frac{\Delta P}{R T L}
$$

where $\varepsilon$ is the porosity and $\mathrm{A}$ is the cross-sectional area of the pore network. Here, $D_{K}=\frac{d_{\text {pore }}}{3} \sqrt{\frac{8 R T}{\pi M}}$ is the Knudsen diffusivity coefficient, which is a function of the characteristic pore diameter $d_{\text {pore }}$, temperature $T$, and the molar mass of gas molecules $M$. The characteristic 
pore diameter $d_{\text {pore }}$, however, cannot be easily determined in sample pore networks. Using the dimensional analysis, we therefore replace $d_{\text {pore }}$ with the reciprocal of the specific surface area (SSA), which is the ratio of surface area $\left([\mathrm{L}]^{2}\right)$ to the pore volume $\left([\mathrm{L}]^{3}\right)$, and calculate the analytical Knudsen diffusivity coefficient in each sample as $D_{K}=\frac{1}{S S A} \sqrt{\frac{8 R T}{\pi M}}$. We assume that the external body force in our simulations is a linear function of the pressure drop across the pore network and thus calculate the numerical Knudsen diffusivity coefficient using Equation 7.

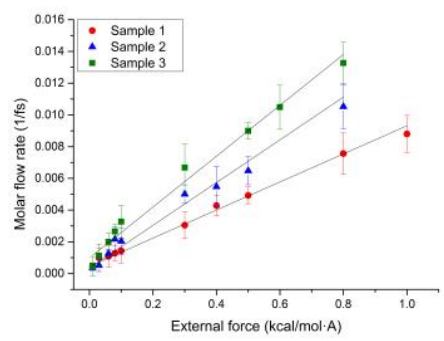

Fig. 11. Average molar flow rate as a function of external body force for each of the three pore networks. The average molar flow rate is the mean molar flow rate in layers 3-8; error bars indicate the standard deviation of the molar flow rate in layers 3-8. Lines are linear fits, indicating that the average molar flow rate is linearly dependent on external body force.

In Equation 7, the slope of the straight line is related to the product of Knudsen diffusivity and geometric parameters of porous media, including porosity and the length of the pore network in the flow direction. The fit parameters extracted from the data in Fig. 11 and the corresponding Knudsen diffusivities are shown in Table 7. The numerical and analytical Knudsen diffusivity coefficients are of the same order of magnitude, despite differences in porosity and surface area. We find that the numerical diffusivity is lower than the analytical diffusivity, and hence effectiveness factor $\eta<1$ as the ratio of the numerical diffusivity to the analytical diffusivity. This result indicates that gas transport through the realistic pore network is not simply a function of specific surface area or characteristic pore diameter. More factors, such as tortuosity or pore connectivity, contribute to the "non-ideal" diffusivity coefficient in diffusion-dominated flow 
and hinder the gas transport through nanoporous media. In the three pore networks, we typically observe that the diffusivity coefficient decreases as the surface area increases in the three pore networks. The molecule-boundary interactions may differ from the elastic interactions assumed in Knudsen flow. Methane molecules can adsorb to neighboring carbon atoms and this interaction can alter their trajectories and hence modify the transport of methane through the network. As the surface area increases, the effectiveness factor decreases, which suggests that the non-ideality of Knudsen diffusion flow becomes predominant.

Table 7. Slope and intercept for linear fits to the data in Fig. 8 and corresponding Knudsen diffusivity coefficients based on Equation 7.

\begin{tabular}{cccccc}
\hline Sample & Slope $\left(\mathrm{k} \times 10^{-3}\right)$ & $\mathrm{R}^{2}$ & $\begin{array}{c}\mathrm{D}_{\mathrm{K}}\left(\times 10^{-7} \mathrm{~m}^{2} / \mathrm{s}\right)^{a} \\
(\text { numerical })\end{array}$ & $\begin{array}{c}\mathrm{D}_{\mathrm{K}}\left(\times 10^{-7} \mathrm{~m}^{2} / \mathrm{s}\right)^{b} \\
(\text { analytical })\end{array}$ & $\begin{array}{c}\text { Effectiveness } \\
\text { factor } \eta^{c}\end{array}$ \\
1 & $8.8 \pm 0.2$ & 0.9937 & $1.57 \pm 0.03$ & 1.96 & 0.80 \\
2 & $13.5 \pm 0.8$ & 0.9735 & $1.07 \pm 0.06$ & 1.98 & 0.54 \\
3 & $16.0 \pm 0.7$ & 0.9853 & $0.52 \pm 0.02$ & 2.31 & 0.23
\end{tabular}

${ }^{a}$ Numerical diffusivity coefficient is calculated from $\left(D_{K}=(k R T L) / n \varepsilon\right)$. Here, we assume the pressure drop is linearly proportional to the external driving force $\left(\Delta P=n F_{E} / A\right)$, where $A$ is the cross-sectional area, $n$ is the total number of methane molecules to which the external force is applied.(Turgman-Cohen et al., 2013; Zhu et al., 2002) In our simulation, two methane molecules are inserted in each pore voxel so that $n$ is equal to 200,300 , and 470 in samples $1-3$, respectively.

${ }^{b}$ Analytical Knudsen diffusivity coefficient is calculated as $D_{K}=\frac{1}{S S A} \sqrt{\frac{8 R T}{\pi M}}$. Here, the specific surface areas of three pore networks are $3.22(322 / 100) \mathrm{nm}^{-1}, 3.17(476 / 150) \mathrm{nm}^{-1}$, and $2.72(640 / 235) \mathrm{nm}^{-1}$, respectively. $M=16.04$ $\mathrm{g} / \mathrm{mol}$ is the molar mass of methane.

${ }^{c}$ Effectiveness factor is taken as the ratio of numerical diffusivity coefficient to the analytical diffusivity coefficient. 


\section{CONCLUSIONS}

Natural gas transport and storage in carbon-based nanoporous networks, determined using the MCMC method, were investigated using a combination of molecular simulation techniques. Digital rock reconstructions were obtained from 2-D SEM images using the MCMC 3-D digital rock reconstruction algorithm. Subsequently, methane storage and transport were characterized in three pore networks of varying porosity and surface area obtained from one digital rock sample. Using Monte Carlo simulations, adsorption isotherms of methane were calculated for temperatures ranging from $300-420 \mathrm{~K}$. The adsorption obeys the Langmuir isotherm, and the equilibrium constants are similar in the three pore networks because only the methane-carbon interaction is considered in the simulation. Non-equilibrium molecular dynamics simulations reveal that molecular diffusion dominates gas transport in quiescent conditions or for weak external body forces. The magnitude of fluctuations of the molar flow rate in the network monotonically decreases as the external body force increases. The transport in nanoscale pore networks displays Knudsen-type diffusion characteristics. The observed linear relationship between the average molar flow rate and external body force is related to the Knudsen diffusivity. Surface area is a major factor that determines the Knudsen diffusivity. As the surface area increases, the diffusivity coefficient decreases as well as the effectiveness factor. More parameters such as pore connectivity and tortuosity may also affect the gas transport through the porous network.

The combination of the MCMC and MD methods offers a unique approach to investigating gas transport in nanoporous media. By capturing increasingly realistic features of shale gas reservoirs, this approach can generate an improved understanding of gas transport properties that 
impacts production performance and reserve estimation, for example, by determining the gas diffusivity through the organic matrix in shale or improving the estimation of methane adsorption by understanding the complicated molecular interactions between different components. In future work, we plan to extend these methods to larger-scale molecular simulations in microscopic pore networks. Our current work simulates organic matter using only carbon atoms as an initial demonstration of this combined simulation approach. In future studies, we will incorporate more realistic molecular structures, such as kerogen, clay, and quartz to identify gas transport and storage mechanisms in shale gas reservoir systems.

\section{ACKNOWLEDGMENTS}

This research is supported in part by US National Science Foundation through NSF Grant DMS1209124, Society of Petroleum Engineers - Gulf Coast Section through its Distinguished

Professorship endowment, Institute of Petroleum Exploration \& Development - Langfang Branch, PetroChina Company Limited, and CNPC Chuanqing Drilling Engineering Company Limited. The authors also thank PetroChina Company Limited for the permission to publish this paper.

We thank Prof. Yao, Jun in China University of Petroleum for access to the Hitachi SEM on which SEM micrographs of shale rock were acquired.

\section{REFERENCES}

Ambrose, R.J., Hartman, R.C., Diaz-Campos, M., Akkutlu, I.Y., Sondergeld, C.H., 2010. New pore-scale considerations for shale gas in place calculations. SPE Unconv. Gas Conf. 2010 167-183. doi:10.2118/131772-MS

Atkins, P., de Paula, J., 2006. Physical Chemistry. W. H. Freeman. 
Berendsen, H.J.C., Postma, J.P.M., van Gunsteren, W.F., DiNola, a., Haak, J.R., 1984. Molecular dynamics with coupling to an external bath. J. Chem. Phys. 81, 3684. doi:10.1063/1.448118

Boek, E.S., Venturoli, M., 2010. Lattice-Boltzmann studies of fluid flow in porous media with realistic rock geometries. Comput. Math. with Appl. 59, 2305-2314. doi:10.1016/j.camwa.2009.08.063

Boţan, A., Ulm, F.-J., Pellenq, R.J.-M., Coasne, B., 2015. Bottom-up model of adsorption and transport in multiscale porous media. Phys. Rev. E 91, 1-10. doi:10.1103/PhysRevE.91.032133

Boțan, A., Vermorel, R., Ulm, F.-J., Pellenq, R.J.-M., 2013. Molecular simulations of supercritical fluid permeation through disordered microporous carbons. Langmuir 29, 9985-90. doi:10.1021/la402087r

Chapman, S., Cowling, T.G., 1970. The Mathematical Theory of Non-uniform Gases: An Account of the Kinetic Theory of Viscosity, Thermal Conduction and Diffusion in Gases, Cambridge Mathematical Library. Cambridge University Press.

Chen, X., Cao, G., Han, A., Punyamurtula, V.K., Liu, L., Culligan, P.J., Kim, T., Qiao, Y., 2008. Nanoscale fluid transport: size and rate effects. Nano Lett. 8, 2988-92. doi: $10.1021 / \mathrm{n} 1802046 \mathrm{~b}$

Civan, F., 2010. Effective correlation of apparent gas permeability in tight porous media. Transp. Porous Media 82, 375-384. doi:10.1007/s11242-009-9432-z

Collell, J., Galliero, G., Gouth, F., Montel, F., Pujol, M., Ungerer, P., Yiannourakou, M., 2014. Molecular simulation and modelisation of methane/ethane mixtures adsorption onto a microporous molecular model of kerogen under typical reservoir conditions. Microporous Mesoporous Mater. 197, 194-203. doi:10.1016/j.micromeso.2014.06.016

Darabi, H., Ettehad, A., Javadpour, F., Sepehrnoori, K., 2012. Gas flow in ultra-tight shale strata 641-658. doi:10.1017/jfm.2012.424

Fathi, E., Akkutlu, I.Y., Texas, A., 2013. Lattice Boltzmann Method for Simulation of Shale Gas Transport in Kerogen 27-37.

Firouzi, M., Wilcox, J., 2012. Molecular modeling of carbon dioxide transport and storage in porous carbon-based materials. Microporous Mesoporous Mater. 158, 195-203. doi:10.1016/j.micromeso.2012.02.045

Frenkel, D., Smit, B., 2002. Understanding of Molecular Simulation: from Algorithms to Applications, Understanding Molecular Simulation. doi:10.1016/B978-012267351$1 / 50005-5$ 
Geman, S., Geman, D., 1984. Stochastic Relaxation, Gibbs Distributions, and the Bayesian Restoration of Images. IEEE Trans. Pattern Anal. Mach. Intell. PAMI-6, 721-741. doi:10.1109/TPAMI.1984.4767596

Heller, R., Zoback, M., 2014. Adsorption of methane and carbon dioxide on gas shale and pure mineral samples. J. Unconv. Oil Gas Resour. 8, 14-24. doi:http://dx.doi.org/10.1016/j.juogr.2014.06.001

Holt, J.K., Park, H.G., Wang, Y., Stadermann, M., Artyukhin, A.B., Grigoropoulos, C.P., Noy, A., Bakajin, O., 2006. Sub - 2-Nanometer Carbon Nanotubes 312, 1034-1037.

Javadpour, F., 2009. Nanopores and apparent permeability of gas flow in mudrocks (shales and siltstone). J. Can. Pet. Technol. 48, 16-21. doi:10.2118/09-08-16-DA

Ji, L., Zhang, T., Milliken, K.L., Qu, J., Zhang, X., 2012. Experimental investigation of main controls to methane adsorption in clay-rich rocks. Appl. Geochemistry 27, 2533-2545. doi:10.1016/j.apgeochem.2012.08.027

Kannam, S.K., Todd, B.D., Hansen, J.S., Daivis, P.J., 2011. Slip flow in graphene nanochannels. J. Chem. Phys. 135, 144701. doi:10.1063/1.3648049

Katti, D.R., Upadhyay, H.B., Katti, K.S., 2014. Molecular interactions of kerogen moieties with Na-montmorillonite: An experimental and modeling study. Fuel 130, 34-45. doi:10.1016/j.fuel.2014.04.009

Li, Y., LeBoeuf, E.J., Basu, P.K., Mahadevan, S., 2005. Stochastic modeling of the permeability of randomly generated porous media. Adv. Water Resour. 28, 835-844. doi:10.1016/j.advwatres.2005.01.007

Majumder, M., Chopra, N., Andrews, R., Hinds, B.J., 2005. Nanoscale hydrodynamics: enhanced flow in carbon nanotubes. Nature 438, 44. doi:10.1038/438930b

Manwart, C., Aaltosalmi, U., Koponen, a., Hilfer, R., Timonen, J., 2002. Lattice-Boltzmann and finite-difference simulations for the permeability for three-dimensional porous media. Phys. Rev. E - Stat. Nonlinear, Soft Matter Phys. 66, 1-32. doi:10.1103/PhysRevE.66.016702

Martin, M.G., Siepmann, J.I., 1998. Transferable Potentials for Phase Equilibria. 1. United-Atom Description of n -Alkanes. J. Phys. Chem. B 102, 2569-2577. doi:10.1021/jp972543+

Mosher, K., He, J., Liu, Y., Rupp, E., Wilcox, J., 2013. Molecular simulation of methane adsorption in micro- and mesoporous carbons with applications to coal and gas shale systems. Int. J. Coal Geol. 109-110, 36-44. doi:10.1016/j.coal.2013.01.001

Orendt, A.M., Pimienta, I.S.O., Badu, S.R., Solum, M.S., Pugmire, R.J., Facelli, J.C., Locke, D.R., Chapman, K.W., Chupas, P.J., Winans, R.E., 2013. Three-Dimensional Structure of 
the Siskin Green River Oil Shale Kerogen Model: A Comparison between Calculated and Observed Properties. Energy \& Fuels 27, 702-710. doi:10.1021/ef3017046

Plimpton, S., 1995. Fast Parallel Algorithms for Short-Range Molecular Dynamics. J. Comput. Phys. doi:10.1006/jcph.1995.1039

Rexer, T.F., Mathia, E.J., Aplin, A.C., Thomas, K.M., 2014. High-Pressure Methane Adsorption and Characterization of Pores in Posidonia Shales and Isolated Kerogens. Energy \& Fuels 28, 2886-2901. doi:10.1021/ef402466m

Roy, S., Raju, R., Chuang, H.F., Cruden, B. a., Meyyappan, M., 2003. Modeling gas flow through microchannels and nanopores. J. Appl. Phys. 93, 4870. doi:10.1063/1.1559936

Sakhaee-Pour, a., Bryant, S.L., 2012. Gas permeability of shale. SPE Reserv. Eval. Eng. 15, 401-409. doi:10.2118/146944-ms

Swami, V., Settari, A.T., 2012. SPE 155756 A Pore Scale Gas Flow Model for Shale Gas Reservoir 5-7.

Thomas, J., McGaughey, A., 2009. Water Flow in Carbon Nanotubes: Transition to Subcontinuum Transport. Phys. Rev. Lett. 102, 184502.

doi:10.1103/PhysRevLett.102.184502

Turgman-Cohen, S., Araque, J.C., Hoek, E.M. V, Escobedo, F. a., 2013. Molecular dynamics of equilibrium and pressure-driven transport properties of water through LTA-type zeolites. Langmuir 29, 12389-12399. doi:10.1021/la402895h

Wang, L., Dumont, R.S., Dickson, J.M., 2013. Nonequilibrium molecular dynamics simulation of pressure-driven water transport through modified CNT membranes. J. Chem. Phys. 138, 124701. doi:10.1063/1.4794685

Wang, L., Dumont, R.S., Dickson, J.M., 2012. Nonequilibrium molecular dynamics simulation of water transport through carbon nanotube membranes at low pressure. J. Chem. Phys. 137, 044102. doi:10.1063/1.4734484

Wang, M., Pan, N., 2008. Predictions of effective physical properties of complex multiphase materials. Mater. Sci. Eng. R Reports 63, 1-30. doi:10.1016/j.mser.2008.07.001

Wu, K., Nunan, N., Crawford, J.W., Young, I.M., Ritz, K., 2004. An Efficient Markov Chain Model for the Simulation of Heterogeneous Soil Structure 346-351.

Wu, K., Van Dijke, M.I.J., Couples, G.D., Jiang, Z., Ma, J., Sorbie, K.S., Crawford, J., Young, I., Zhang, X., 2006. 3D Stochastic Modelling of Heterogeneous Porous Media - Applications to Reservoir Rocks. Transp. Porous Media 65, 443-467. doi:10.1007/s11242-006-0006-Z 
Yang, A., Miller, C.T., Turcoliver, L.D., 1996. Simulation of correlated and uncorrelated packing of random size spheres. Phys. Rev. E 53, 1516-1524.

doi:10.1103/PhysRevE.53.1516

Yao, J., Wang, C., Yang, Y., Hu, R., Wang, X., 2013. The construction of carbonate digital rock with hybrid superposition method. J. Pet. Sci. Eng. 110, 263-267.

doi:10.1016/j.petrol.2013.10.005

Yiannourakou, M., Ungerer, P., Leblanc, B., Rozanska, X., Saxe, P., Vidal-Gilbert, S., Gouth, F., Montel, F., 2013. Molecular Simulation of Adsorption in Microporous Materials. Oil Gas

Sci. Technol. - Rev. d'IFP Energies Nouv. 68, 977-994. doi:10.2516/ogst/2013134

Yuan, W., Pan, Z., Li, X., Yang, Y., Zhao, C., Connell, L.D., Li, S., He, J., 2014. Experimental study and modelling of methane adsorption and diffusion in shale. Fuel 117, 509-519. doi:10.1016/j.fuel.2013.09.046

Zhang, T., Ellis, G.S., Ruppel, S.C., Milliken, K., Yang, R., 2012. Effect of organic-matter type and thermal maturity on methane adsorption in shale-gas systems. Org. Geochem. 47, 120131. doi:10.1016/j.orggeochem.2012.03.012

Zhao, X., Yao, J., Yi, Y., 2006. A new stochastic method of reconstructing porous media. Transp. Porous Media 69, 1-11. doi:10.1007/s11242-006-9052-9

Zheng, M., Li, X., Liu, J., Wang, Z., Gong, X., Guo, L., Song, W., 2014. Pyrolysis of liulin coal simulated by GPU-based ReaxFF MD with cheminformatics analysis. Energy and Fuels 28, 522-534. doi:10.1021/ef402140n

Zhu, F., Tajkhorshid, E., Schulten, K., 2002. Pressure-induced water transport in membrane channels studied by molecular dynamics. Biophys. J. 83, 154-160. doi:10.1016/S00063495(02)75157-6

\section{Highlights:}

In this paper, we have applied molecular simulation to study the mechanisms of natural gas storage and transport in complex nano-scale pore structures. The highlights of this work are as follows:

1. Grand canonical Monte Carlo simulation is applied to study the mechanisms of natural gas storage behavior and non-equilibrium molecular dynamics simulation is applied to investigate the gas transport behavior in complex nano-scale pore structure.

2. Complex nano-pore structures are reconstructed by digital rock reconstruction technique, Markov Chain Monte Carlo Simulation, based on the 2-D scanning electron microscopy (SEM) images of a real shale rock sample.

3. Preliminary results show the complex geometry of pore structure, such as tortuosity, surface area, etc. play important roles in gas storage and transport behaviors. 